\title{
Distinct patterns of word retrieval in right and left frontal lobe patients: a multidimensional perspective
}

\author{
Sophie Schwartz ${ }^{\mathrm{a}, *}$, Juliana Baldo ${ }^{\mathrm{b}}$ \\ ${ }^{a}$ Institute of Cognitive Neuroscience (ICN), University College London, Alexandra House, 17 Queen Square, London WC1N 3AR, UK \\ ${ }^{\mathrm{b}}$ VA Northern California Health Care System, Martinez, CA, USA
}

Received 15 May 2000; received in revised form 3 February 2001; accepted 7 March 2001

\begin{abstract}
Several studies have shown that patients with frontal lobe damage (FL) generate fewer words than control participants in verbal fluency tasks. In the current study, we were interested in assessing the quality of such verbal fluency data. Multidimensional exploratory techniques, namely, hierarchical clustering (HC) and correspondence analysis (CoA), were performed on verbal fluency data collected from $13 \mathrm{FL}$ patients and 11 age-matched controls. $\mathrm{HC}$ of individual word-profiles distinguished between patients with left frontal lobe (LFL) and right frontal lobe (RFL) lesions. CoA revealed that LFL patients generated unusual word-profiles, as evidenced by their peripheral location on a CoA map, while RFL patients produced highly typical word-profiles. We would like to suggest that preserved functioning of the left frontal lobe might have constrained the responses of the RFL group resulting in typical word combinations, whereas preserved right frontal lobe functioning might have mediated greater diversification in the responses of the LFL group. The present study shows that HC and CoA can be applied to neuropsychological data to reveal complex relationships between cognitive performance and cerebral lesion sites. (C) 2001 Elsevier Science Ltd. All rights reserved.
\end{abstract}

Keywords: Correspondence analysis; Clustering; Executive functions; Cognitive maps; Laterality

\section{Introduction}

Verbal fluency tasks have been used as a measure of 'executive' function in patients with brain injury [24,27]. These tasks require patients to generate as many words as they can in a given time period (usually $1-2 \mathrm{~min}$ ). Patients may be asked to generate items belonging to one specific category, for example, 'animals' (category fluency), or words beginning with a certain letter (letter or phonemic fluency). This ability to search through memory and retrieve exemplars is thought to reflect executive processes and has been associated with the frontal lobes $[5,24]$. Traditionally, the critical measure in verbal fluency tasks is the number of items generated by participants. However, little research has been done to analyze which words are generated, i.e. the quality of responses in fluency tasks. In timed, short tasks such as

\footnotetext{
* Corresponding author. Tel.: +44-20-76791127; fax: +44-2079168517.

E-mail address: s.schwartz@ucl.ac.uk (S. Schwartz).
}

verbal fluency, the participant's choice of words might reveal interesting features of his/her semantic network or semantic search strategies.

A few recent studies have analyzed fluency responses in order to assess the respective contributions of both associational and executive components. In fluency tasks, successive associations tend to occur over time in sequences of semantically or phonemically related words [18]. It has been postulated that this sequential grouping may relate to temporal-lobe processes, such as semantic verbal memory. Switching between groups of related concepts has been found to rely on frontal-lobe processes such as strategic search, cognitive flexibility, and attentional monitoring [32,33]. Optimal performance probably requires a subtle interaction between temporal-lobe associative grouping and frontal switching [33].

More specific assessments of the structure of semantic knowledge have been done using multidimensional techniques on fluency data, by placing the words gener- 


\begin{tabular}{l|c|c|c|}
\multirow{2}{*}{$\mathbf{a}$} & \multicolumn{3}{|c|}{ words } \\
\cline { 2 - 4 } & cat & tiger & goat \\
\hline subject 1 & 1 & 1 & 0 \\
\hline subject 2 & 1 & 1 & 0 \\
\hline subject 3 & 0 & 1 & 0 \\
\hline subject 4 & 0 & 0 & 1 \\
\hline
\end{tabular}

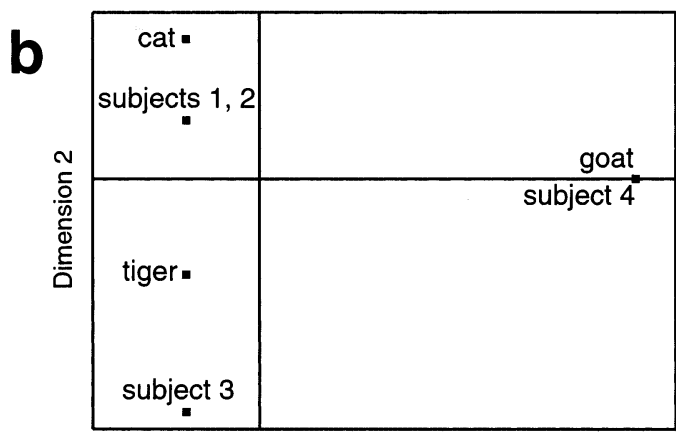

Dimension 1

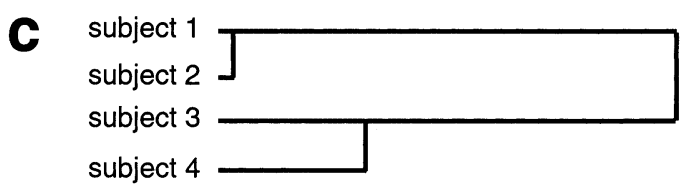

Fig. 1. Visualization of fictive fluency data by CoA and HC. (a) Table with the fluency responses for each individual. (b) CoA display of these data. (c) Hierarchical tree corresponding to the successive clustering of the most similar individual responses, from the left to the right.

ated into a coordinate space forming a 'cognitive map' $[1,10]$. The resulting maps have been interpreted on the basis of the following principle proposed by spreadingactivation theories: When a given concept or node in a network is activated, the level of activation of related concepts increases $[2,12]$. It follows that two strongly related concepts would be produced within a close temporal distance in fluency tasks and would also be rated as similar. Accordingly, distances between items as displayed on cognitive maps should reflect psychological distances (e.g. 'dog' and 'cat' would be close, while 'dog' and 'penguin' would be distant). For example, using multidimensional scaling (MDS) on fluency data, Chan et al. [10] found that semantic networks of patients with Alzheimer's disease were poorly organized, compared to normal control participants and patients with Huntington's disease. This finding is consistent with the notion that semantic organization depends upon the integrity of the temporal lobes [31].

There are methodological limitations with using MDS to analyze fluency data, however. For fluency tasks (unlike comparison tasks, see below), semantic distances between words are estimated from the number of items separating each pair of words within fluency responses. The reliability of the resulting similarity matrices is questionable because each participant does not generate all the items whose distances are estimated [28]. Also, the large variance in the number of items provided by each participant may limit the use of MDS for assessing individual differences in fluency responses. Furthermore, MDS techniques are based on several metric hypotheses that are often violated by psychological data [34]. In particular, while MDS maps reflect the sequential distances between words within fluency responses, MDS does not take into account the chrono- logical order in which the words have been generated (symmetry hypothesis); however, associative links between words are usually asymmetric. For example, Marshall and Cofer [22] found that the word 'horse' is a common associate of the word 'zebra' (13/100 of the first associates), while conversely the word 'zebra' is not a common associate of the word 'horse' $(0 / 100$ of the first associates). Therefore, it might be preferable not to use sequential distances between pairs of words when using multidimensional procedures with fluency data.

Here, we postulate that in short, timed tasks like verbal fluency, participants generate words that are the most quickly accessed in their semantic networks. This implies that each participant could possibly be better characterized by the particular combination of words that he or she produced than by the order in which he or she produced a subset of selected words. We chose to use correspondence analysis (CoA) and hierarchical clustering techniques (HC) as alternative multidimensional tools that precisely allow one to compare fluency responses according to which words they contain, revealing patterns of semantic accessibility rather than a geometric solution for sequential distances between pairs of words [6,17].

$\mathrm{CoA}$ is a multivariate method for exploring tables of numerical information. CoA converts such tables into graphical displays, called maps, and provides related numerical statistics. Input data to $\mathrm{CoA}$ is usually a matrix of raw frequencies. This technique can easily be applied to a table summarizing fluency data collected from different individuals. Such a table would have as many rows as there are participants and as many columns as the number of words generated by all the individuals (Fig. 1). Geometrical distances on a $\mathrm{CoA}$ map reflect the resemblance between rows (or the re- 
semblance between columns) within the contingency table $^{1}$. In addition, it is possible to cluster the participants' word-profiles without any a priori grouping of their responses, using hierarchical clustering techniques (HC) $[15,20]$. Thus, HC can complement the results derived from CoA.

An example of these procedures is shown in Fig. 1. In this simplified example, two participants said 'cat' and 'tiger,' a third participant only said 'tiger,' and a fourth participant only said 'goat.' Fig. 1 shows the table crossing the four participants with the three possible words and the CoA map derived from the table. Along the first, main dimension of the CoA map, participant 4 is distinguished from the three other participants that are gathered on the left side of the map. The second dimension further distinguishes participants 1 and 2 from participant 3. Fig. 1 also shows the grouping of the participants by $\mathrm{HC}$ where participants 1 and 2 are first grouped together, while participants 3 and 4 form a distinct group.

CoA maps of fluency data can plot the proximities between individuals or groups as well as the proximities among words. As shown in this example, responses that include similar combinations of words would be close to each other on a CoA map, as would individual words that are frequently generated together. It is a remarkable property of $\mathrm{CoA}$ and $\mathrm{HC}$ that each individual response is defined by both the presence as well as the absence of any given word. Two advantages of CoA and $\mathrm{HC}$ are that these techniques are simple and make the fewest possible assumptions about the underlying distribution of the data $[6,7,17]$.

Comparing word-profiles involves a much larger amount of data per participant than when using other multidimensional procedures, in which similarity matrices are based on the number of words separating pairs of words. This issue is particularly relevant when analyzing data from patients whose fluency performance might be very poor or atypical. Moreover, since proximities between CoA profiles represent similarities between fluency responses considered as ensembles of words rather than similarities in the order in which

\footnotetext{
${ }^{1}$ To derive a CoA map from a contingency table, each row (or each column) is reduced to a common base of $100 \%$ by computing percentages relative to the rows (or columns) totals. Such a set of percentages, calculated for a row (or a column) of frequencies, is called a profile. Profiles have a geometric interpretation since they define points in a multidimensional space; they are examples of mathematical 'vectors'. CoA uses a variation of Euclidian distance called a weighted Euclidian distance to measure geometric distances between profile points within the multidimensional space. The goal of CoA is to select a low-dimensional space, usually a plane, which reflects as accurately as possible the distances between the profiles. This is done by looking for the plane which is 'closest', in terms of weighted least-squares, to all the points (see Ref. [17] for a didactic introduction to CoA principles; about how to compute CoA, see the SPSS Categories Manual, SPSS, Chicago, IL).
}

words are generated, asymmetry in associative links should not significantly affect the results. Thus, CoA provides crucial information about lexico-semantic processing by reflecting the likelihood of two or more words co-occurring in the same responses. Given that fluency tasks are performed under time pressure, words generated under such constraints are those most readily accessed.

In the present study, we used $\mathrm{HC}$ and $\mathrm{CoA}$ to determine whether frontal patients' (FL) word-profiles exhibit individual differences in search strategies and monitoring of lexico-semantic information. Several lines of evidence suggest that patterns of semantic associations are differentially monitored by the left and right frontal lobes. For example, priming experiments indicate that the left hemisphere inhibits remote word associations, while the right hemisphere provides a longer-lasting activation of both strongly-related and distantly-related words [11,26]. Right frontal regions have also been implicated in non-routine cognitive situations requiring innovative behavior, whereas left frontal regions have been shown to allow a rapid access to pre-existing representations while inhibiting irrelevant information [14,16]. Accordingly, we expected that frontal patients with left-sided lesions would generate more unusual combinations of words compared to patients with lesions in the right frontal regions. We tested this hypothesis using $\mathrm{HC}$ and $\mathrm{CoA}$, and showed that these multidimensional procedures can be used to reveal specific qualitative patterns in verbal fluency data collected from patients with frontal lobe lesions. Conventional analyses of these verbal fluency data, along with data from a design fluency task, are reported elsewhere [4]. To our knowledge, this is the first time that $\mathrm{CoA}$ has been applied to fluency data.

\section{Method}

\subsection{Participants}

Thirteen patients (nine male, four female) with focal frontal lobe lesions were recruited for this study (Table 1). Patients with lesions extending into non-frontal regions were excluded. Most of the patients had lesions localized to lateral prefrontal cortex: six of these patients had focal left hemisphere lesions, and five had focal right hemisphere lesions, and two patients had bilateral, orbital frontal lesions. The latter were included in order to obtain a corpus of words that was large enough for a valid CoA. In seven of the patients, lesions were due to an infarct of the anterior branch of the middle cerebral artery. In the other patients, lesions were due to surgical treatment for an aneurysm, arterial-venous malformation, cyst, or meningioma. Patients had an average lesion volume of $53.5 \mathrm{cc}$ (Table 
Table 1

Participant characterization ${ }^{\mathrm{a}}$

\begin{tabular}{|c|c|c|c|c|c|c|c|c|}
\hline Patient & Gender & Lesion site & Volume (cc) & Age & $\begin{array}{l}\text { Years } \\
\text { post-onset }\end{array}$ & Lesion etiology & $\begin{array}{l}\text { Education } \\
\text { (years) }\end{array}$ & WAIS-RPIQ \\
\hline $\mathrm{OA}$ & M & $\mathrm{L}$ & 17.5 & 62 & 13 & Stroke & 14 & 134 \\
\hline EB & $\mathrm{F}$ & $\mathrm{R}$ & 17.3 & 77 & 14 & Stroke & 12 & 114 \\
\hline SR & $\mathrm{F}$ & $\mathrm{R}$ & 12.9 & 76 & 2 & Stroke & 12 & 93 \\
\hline WA & $\mathrm{F}$ & $\mathrm{L}$ & 26.2 & 74 & 11 & Stroke & 14 & 132 \\
\hline WT & M & $\mathrm{R}$ & 25.9 & 52 & 9 & Cyst & 18 & - \\
\hline MG & M & $\mathrm{R}$ & 24.5 & 33 & 13 & AVM & 12 & - \\
\hline MK & M & $\mathrm{R}$ & 200.4 & 65 & 18 & Aneurysm & 17 & - \\
\hline LS & $\mathrm{F}$ & $\mathrm{L}$ & 27.9 & 67 & 16 & Mening. & 16 & - \\
\hline $\mathrm{CL}$ & M & R-L & - & 64 & 10 & Mening. & 16 & - \\
\hline WP & M & R-L & - & 54 & 0.08 & Mening. & 12 & - \\
\hline FE & $\mathrm{M}$ & $\mathrm{L}$ & 41.1 & 68 & 2 & Stroke & 14 & 104 \\
\hline $\mathrm{JC}$ & M & $\mathrm{L}$ & 102.6 & 73 & 10 & Stroke & 16 & 103 \\
\hline WR & M & $\mathrm{L}$ & 91.6 & 55 & 7 & Stroke & 15 & 63 \\
\hline \multicolumn{9}{|l|}{ Means } \\
\hline Frontal & $9 \mathrm{M}, 4 \mathrm{~F}$ & $6 \mathrm{~L}, 5 \mathrm{R}$ & $53.45(57.32)$ & $63.08(12.24)$ & $9.62(5.56)$ & - & $14.46(2.07)$ & 106.14 \\
\hline Control & $8 \mathrm{M}, 3 \mathrm{~F}$ & - & - & $68.03(5.76)$ & - & - & $14.82(2.32)$ & - \\
\hline
\end{tabular}

${ }^{a}$ Dashes indicate data that are not available or not applicable. L, left hemisphere; R, right hemisphere; R-L, bilateral orbital frontal; AVM, arterio-venous malformation; Mening., meningioma (removed); WAIS-RPIQ, Wechsler Adult Intelligence Scale Revised Performance IQ.

1). Patients were tested in the chronic stage of their injury ( $>1$ year post-onset) except one patient (WP). The average time since onset of injury was 9.6 years. Patients were in the normal range on the Western Aphasia Battery [19], except for one mildly aphasic patient (Aphasia Quotient or AQ of 90/100, where 93.7 is normal), and another patient with moderate aphasia $(A Q=76 / 100)$. Both of these patients had relatively intact comprehension. These frontal lobe patients were not excluded, because their fluency scores were within two standard deviations of patients' mean scores. All patients were premorbidly right-handed.

The control group consisted of 11 healthy controls (eight men and three women) recruited from the same community as the patients. Controls were all righthanded. Patients and controls did not differ in terms of age $(M=63.1$ and 68.0 , respectively; $F(1,22)=1.55$, $P=23)$ or education $(M=14.6$ and 14.8 , respectively; $F(1,22)=1.59, P=0.69)$. Testing was conducted at the Veterans Administration Northern California Health Care System in Martinez, CA. All patients and controls were screened for history of dementia, drug abuse, and psychiatric illness, and controls were additionally screened for prior neurological history. All participants spoke English as their first language. Participants read and signed consent forms prior to participating in the study.

\subsection{Procedure}

A category fluency task was administered individually to participants, as part of a neuropsychological battery. Participants were given $60 \mathrm{~s}$ to produce as many names of animals as possible. They were told that they could name any type of animal. The examiner recorded participants' responses.

Hierarchical clustering ( $\mathrm{HC})$ and correspondence analysis (CoA) were computed, using the 25 most frequently generated words in the total sample (i.e. control participants and FL patients). The following 25 words occurred at least five times in the total sample (listed by decreasing total frequency): dog, cat, lion, tiger, elephant, giraffe, bear, cow, horse, zebra, fox, monkey, rat, alligator, mouse, pig, rabbit, snake, chicken, deer, eagle, goat, leopard, sheep, squirrel. The choice of this range of frequency was motivated by the fact that a sufficient number of co-occurrences of words is needed within each fluency response in order to cluster individual word-profiles.

As a preliminary step to conducting $\mathrm{HC}$ and $\mathrm{CoA}$, fluency responses were organized into a contingency table. The 25 most frequent words produced constituted the columns of this table, while the 24 participants' responses or individual word-profiles formed the rows of the table (cf. Fig. 1a). This table provided the basis for subsequent $\mathrm{HC}$ and $\mathrm{CoA}$.

$\mathrm{HC}$ was first computed in order to form groups of participants who generated similar word-profiles [20]. $\mathrm{HC}$ was performed using measures of similarities that can be applied to contingency tables and thus enabled us to make the resulting clusters compatible with CoA factorial scores. Estimation of the distance or similarity between two cases (rows or columns) in the contingency table was based on a $\chi^{2}$-statistic. Formation of clusters was done following a hierarchical method (Ward method, see [20] for details). Thus, hierarchical cluster- 
ing performs successive groupings of the data according to their coordinates within the complete initial multidimensional space, unlike CoA maps that show a flattened projection of the data on a continuous space of reduced dimensions (usually a bidimensional space, see below).

CoA was then used to visualize distances between the 24 participants (i.e. their word-profiles) as well as distances between the 25 words. The CoA procedure can be summarized as follows: (1) the row and the column profiles of the contingency table are projected into two different multidimensional spaces, (2) these multidimensional spaces are 'flattened' or reduced onto bidimensional maps that show off the largest amount of inertia, which is a direct measure of the dispersion of the profiles in multidimensional space, and (3) the two maps are convolved into a single conjoint map displaying the row and column profiles together, so that both distances among words and distances among participants are transposed onto the same CoA map [17]. Whereas the CoA procedure produces a conjoint map in which rows and columns of the initial table are organized along continuous factors, $\mathrm{HC}$ results in a discrete grouping of the data. All analyses were performed using SPSS v6.1 (SPSS, Chicago, IL).

\section{Results}

A one-way analysis of variance (ANOVA) was done to compare the total frequency with which the 25 animal names were generated by the control group $(M=11.45$, S.D. $=2.50)$ and the FL-patient group $(M=10.08$, S.D. $=2.72)$. No significant difference was found, $F(1,22)=11.31, P=0.21$, confirming that both groups generated these 25 words with the same frequency. Thus, distances on the CoA should primarily reflect qualitative differences in the word-profiles rather than quantitative differences between the groups. This verification was crucial given that total fluency performance in FL-patients is generally impaired [5]. In our group of patients, the total number of words generated was significantly lower than in the control group $(M=$ 13.92, S.D. $=4.03$ and $M=21.36$, S.D. $=5.07$, respectively), $F(1,22)=16.07, P<0.01$.

HC was computed for the 13 FL-patients' wordprofiles. The first partition created two groups: (1) a cluster of mostly left frontal (LFL) patients (five LFLpatients, one RFL-patient) and (2) a cluster of mostly right frontal (RFL) patients (four RFL-patients, one LFL-patient). The two bilateral orbitofrontal patients were split between the two clusters, thus not biasing the CoA structure. Hereafter, we refer to the cluster with predominantly LFL-patients as the 'LFL-cluster' and the cluster with mostly RFL-patients as the 'RFL-cluster.' This partitioning of patients was significantly dif- ferent from chance, $F(1,11)=4.41, P<0.05$. The LFL- and RFL-clusters did not significantly differ in the total number of words they generated, $(M=12.00$, S.D. $=4.40$ and $M=16.17$, S.D. $=2.14$, respectively), $F$ $(1,11)=4.44, P=0.06$ [5] and, importantly, they did not differ in the number of the 25 animal names they produced $(M=9.57, \quad$ S.D. $=2.44$ and $M=10.67$, S.D. $=3.14$, respectively), $F(1,11)=0.50, P=0.49$. Thus, the two clusters did not merely reflect a quantitative difference in the frequency with which the two groups generated the 25 words.

CoA was conducted on all 24 participants' wordprofiles for the 25 words. Fig. 2 displays the distance between participants as revealed on the plane of the first two principal dimensions of the joint CoA map ${ }^{2}$. The eigen values associated with these two first dimensions were 0.20324 and 0.18565 , accounting for 15.6 and $14.2 \%$ of the total inertia, respectively ${ }^{3}$. On the CoA map, the word-profiles of the LFL- and RFL-cluster showed a striking arrangement, with the LFL-cluster almost surrounding the RFL-cluster. Such a spatial segregation between the two frontal groups contrasts with the control group that is neither confined to a central area nor to a peripheral area of the CoA map. According to CoA principles, the closer a participant's profile is to the axis of one dimension, the more similar the profile is to the mean profile of the entire sample on that dimension [17]. Thus, the RFL-patients' wordprofiles were more similar to the mean profile of the sample on both principal dimensions, compared to LFL-patients' and controls' word-profiles. The control participants' word-profiles did not show any restriction to either the central or peripheral areas of the display.

On the same joint CoA map, the 10 most frequent words (frequency $14-22$ in the total sample) were grouped in a central area of the display (hatched area on Fig. 2), while the 15 moderately frequent words (frequency 5-9) were dispersed outside this central area and distributed among the four quadrants. This array indicates that highly frequent words have a strong probability of being conjointly generated. We examined if the specific spatial arrangement exhibited by the patients resulted from a greater ratio of highly frequent words $(N=10)$ versus moderately frequent words $(N=$

\footnotetext{
${ }^{2}$ The selection of the first two dimensions of the CoA was done according to Cattell's 'scree test', looking at the number of factors preceding a discontinuity in the histogram of the decreasing eigen values [9].

${ }^{3}$ Such percentages of inertia cannot directly be interpreted as percentages of information or variance explained [17]. Using simulation experiments, Lebart et al. [21] have shown that very small percentages of inertia associated with the first two dimensions of a $\mathrm{CoA}$ often correspond to highly specific and stable CoA maps. Moreover, CoA results are particularly resistant when new variables are added [7].
} 
15) in the RFL-cluster compared to the LFL-cluster. The fluency scores for the 25 most frequent words were analyzed in a $2 \times 2$ ANOVA, with Word Frequency Category (highly or moderately frequent) as a withinsubjects factor and Cluster (LFL- vs. RFL-cluster) as a between-subjects factor. There was an overall main effect of Word Frequency Category, $F(1,11)=62.96$, $P<0.0001$, as there were more frequent words produced across both groups of patients. There was no significant main effect of Cluster, demonstrating that LRL- and RFL-clusters generated a comparable number of the 25 frequent words, $F(1,11)=0.50, P=0.49$, as previously shown. More importantly, there was no significant Word Frequency Category $\times$ Cluster interaction, $F(1,9)=4.05, P=0.10$. Thus, the central position of the RFL-cluster was not related to an over-representation of highly frequent words in this group. Rather, the particular geometric organization shown by the CoA map - with the LFL-cluster in the periphery relative to the RFL-cluster - indicates that RFL-patient's word-profiles showed combinations of words that were highly common across the entire sample. Conversely, LFL-patients tended to produce unusual combinations of words.

Finally, we checked for the stability of these results in two ways. First, we re-analyzed patients' and controls' word-profiles with $\mathrm{HC}$ for the 10 most frequent words. The clustering showed the same partition of the sample between LFL- and RFL-clusters, as previously described. Second, a CoA of word-profiles for the same
25 words in the patients alone, without controls (13 patients $\times 25$ words), confirmed the central position of the RFL-patients with the LFL-patients localized exclusively in peripheral locations. The distinct pattern observed in the two groups of patients was thus not due to a distribution imposed by the controls' word-profiles.

\section{Discussion}

In this study, we investigated how frontal lesions might affect verbal fluency performance. Rather than conventional analyses of overall output, we used hierarchical analysis (HC) and correspondence analysis $(\mathrm{CoA})$ to characterize verbal fluency data in a more qualitative manner. The $\mathrm{HC}$ analysis distinguished between two clusters of patients, one consisting of mostly left frontal patients (LFL) and a second consisting of mostly right frontal (RFL) patients. The LFL-cluster exhibited relatively unusual word-profiles, as revealed by their exocentric location on a CoA map. Their absence from the central area of the display suggests that they deviated from dominant combinations of words. In contrast, patients belonging to the RFL-cluster produced more 'prototypic' combinations of words, shown by their being centrally grouped on the CoA map. Taken together, the clustering of the patients' fluency responses as well as their remarkable arrangement on a CoA map indicate that left and right frontal patients differ qualitatively in the way they generate items from a semantic category.

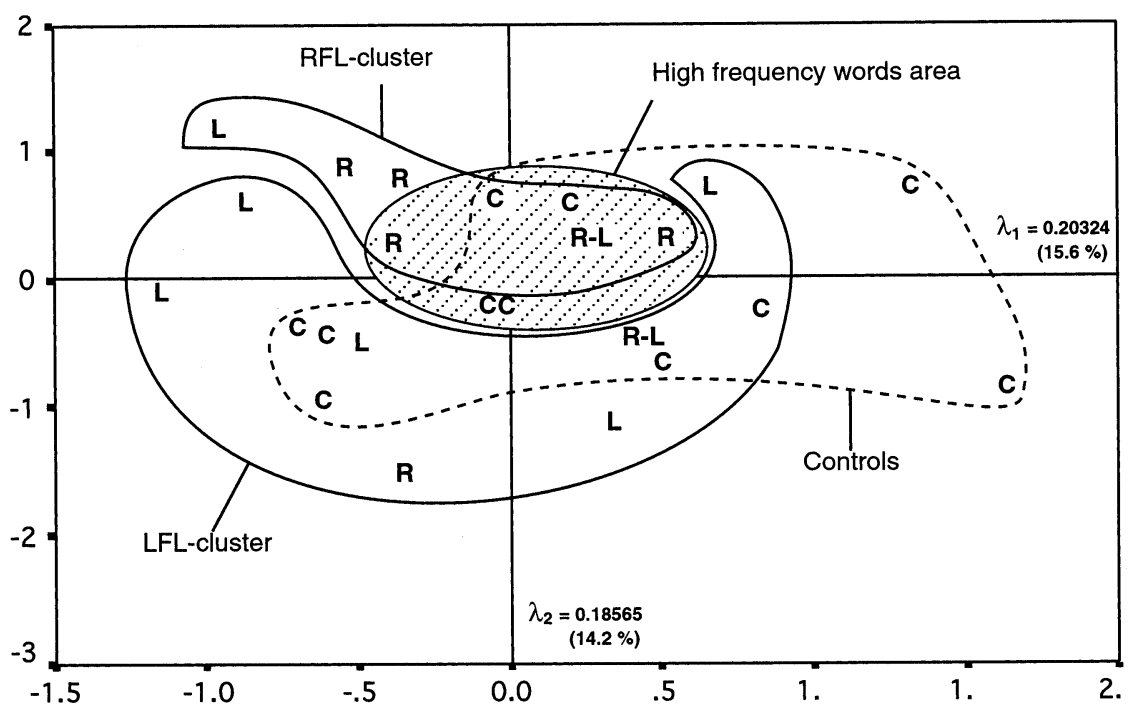

Fig. 2. Two-dimensional CoA conjoint map ( 24 participants $\times 25$ frequent words) showing the spatial arrangement of participants' word-profiles. The letters on the map indicate the position of individual patients, as obtained by CoA (L, LFL patient; R, RFL patient; R-L, patient with bilateral orbital frontal lesion) or controls (C, control). The central hatched area shows the location of the 10 highly frequent words ( $\geq 14$ ), obtained from the same analysis by projecting the word entries instead of the participant entries. Encircled points correspond to the grouping of participants as independently defined by the cluster analysis. This CoA map reveals a remarkable spatial arrangement of the participant groups: the RFL-cluster lies in the central area, the LFL is exclusively located outside this central area, and the control group is evenly dispersed across the display. Eigen values $\left(\lambda_{1}\right.$ and $\left.\lambda_{2}\right)$ and percentages of inertia (reflecting the degree of dispersion of the points projected on each dimension) are displayed along the main axis of the map. 
Using conventional quantitative analyses on the same patients' fluency performance, Baldo et al. [4] found that FL patients were impaired on both verbal and design fluency tasks compared to control participants. The present findings suggest that frontal-lobe processes are not only critical for quantitative productivity in fluency tasks, but also control the access to lexico-semantic knowledge. In particular, while integrity of the left frontal lobe appears to be required for normal quantitative productivity [5], the right frontal lobe is implicated in generating qualitative diversity of the verbal fluency responses [16]. Taken together, the clustering of the patients' fluency responses as well as their remarkable arrangement on a CoA map indicate that left and right frontal patients differ qualitatively in their access to semantic networks.

These findings are consistent with the idea that cerebral hemispheres might participate in different yet complementary aspects of lexico-semantic processing. Namely, left frontal lobe processes appear to be constrained by fixed taxonomic systems, concerned with strong or prototypic semantic connections. In contrast, right frontal lobe processes subserve greater diversity of immediate associations, as if less influenced by the strength of their inter-connections. Previous studies have shown that the left hemisphere is critical for the rapid control and context-based restriction of associative spreading of activation within semantic networks, whereas the right hemisphere activates more remote or indirect associations, independently of category dominance $[11,26,30]$.

It has been further suggested that the left hemisphere is critical for processing based on routinized cognitive strategies, while the right hemisphere supplies exploratory processing of novel cognitive situations, as well as processing of unusual, less frequent, or metaphoric meanings of words $[8,16]$. A role of the right hemisphere in creating novelty is consistent with our data, since patients with left frontal damage generated rather unusual combinations of words. On the other hand, the left hemisphere's advantage in using structured knowledge to rapidly select and monitor adaptive responses is consistent with the prototypical patterns of word-profiles shown by the RFL-cluster. Thus, performance on verbal fluency and other complex tasks may rely on a subtle balance between the explorative attempts of the right hemisphere and conservative tendencies of the left hemisphere.

In addition to frontal-lobe processes that subserve cue-independent executive processes (e.g. the ability to initiate strategic search and retrieval mechanisms [5]), verbal fluency also requires activation of stored semantic representations. These representations are most likely stored in the temporal cortex, as suggested by findings from functional neuroimaging and patient studies [23,25]. Previous studies using MDS have confi- rmed a breakdown of semantic organization in patients with temporal lobe dysfunction, for example, in Alzheimer's disease [10] and schizophrenia [1]. In a pilot study, MDS was performed on similarity judgments produced by a group of $11 \mathrm{FL}$ patients, nine of whom also participated in the present study [3]. Participants were presented with triads of animal names and asked to choose the two animals that they felt "went together.' MDS results did not differ between FL patients and controls suggesting that FL patients might have relatively preserved structure of semantic representations. In contrast, the same FL patients exhibited difficulties in the current study when retrieving animal names in fluency tasks that critically require executive control and monitoring unlike triadic comparison tasks. However, since the number of subjects in the pilot study was quite small, such a negative finding is indicative rather than conclusive of an intact semantic knowledge in these patients. Likewise, we cannot exclude that quantitatively poor fluency performance may be due to disrupted access to lexical knowledge.

While it is generally accepted that participation of both frontal-lobe executive processes and temporal-lobe semantic organization is critical for verbal fluency performance [13,29], the current results of $\mathrm{CoA}$ and $\mathrm{HC}$ performed on data from FL patients suggest that deficits in search strategies and access to semantic knowledge can differ according to the lateralization of the frontal damage. Distinct word-profiles of the two patients' groups might be related to preserved functioning subserved by intact brain regions. In particular, although one must be cautious in using lesion data to understand normal neurocognitive processes, we would like to suggest that preserved functioning of the left frontal lobe might have constrained the responses of the RFL group, whereas preserved right frontal lobe functioning might have mediated a marked diversification in the responses of the LFL group.

There are however some limitations to the current results. First, the etiologies of frontal injury in the current patient group were not uniform, and lesion size varied. Second, the sample sizes were relatively small. Studying larger groups of patients whose brain lesions are more homogeneous would be a necessary step towards generalization of the results. Third, two patients (MK and WR) were misclassified by the HC procedure. MK had a right-sided lesion but was clustered with the LFL group. One possible explanation is that MK was many years post-onset at the time of testing (18 years), which could have led to functional compensation and neural reorganization. WR had a left-sided lesion but was clustered with the RFL group. WR might have failed to show much diversity in his choice of words because of a low PIQ (63) and because he generated few words (seven words while the mean for the patients' group was 13.92, S.D. =4.03). Remarkably, however, 
on the CoA map, WR's word-profile appears quite exocentric relative to the RFL patients, much like the LFL patients' profiles.

The present paper describes a novel way of approaching conventional, neuropsychological data. Namely, the complementary use of hierarchical cluster analysis and correspondence analysis procedures revealed characteristic patterns of performance in a verbal fluency task. We found that left and right frontal patients had distinct patterns of word-profiles, consistent with and extending previous theories of hemispheric specialization. Given that a wide range of verbal and non-verbal neuropsychological data can be summarized into contingency tables crossing two or more nominal variables, multidimensional procedures like $\mathrm{HC}$ and $\mathrm{CoA}$ (or multiple $\mathrm{CoA}$ ) may become very useful in exploring the complex relationships between cerebral lesion sites and patients' performance on different tasks.

\section{Acknowledgements}

This paper benefited greatly from valuable comments of three anonymous reviewers. S. Schwartz was supported by a grant from the Swiss National Science Foundation (grant 8210-061240) and J. Baldo was supported by a National Institutes of Health Research Service Award.

\section{References}

[1] Aloia MS, Gourovitch ML, Weinberger DR, Goldberg TE. An investigation of semantic space in patients with schizophrenia. Journal of the International Neuropsychological Society 1996;2:267-73.

[2] Anderson JR, Pirolli PL. Spread of activation. Journal of Experimental Psychology: Learning, Memory and Cognition 1984;10:791-8.

[3] Baldo J, Chen C-Y, Shimamura A. Impaired memory retrieval in frontal lobe patients, despite intact semantic space. Society for Neuroscience Abstracts 1997;23:1579.

[4] Baldo J, Shimamura A, Delis D, Kramer J, Kaplan E. Verbal and design fluency in patients with frontal lobe lesions. Journal of the International Neuropsychological Society 2001;7:586-96.

[5] Baldo JV, Shimamura AP. Letter and category fluency in patients with frontal lobe lesions. Neuropsychology 1998;12:25967.

[6] Benzécri J-P. L’analyse des données. Paris: Dunod, 1973.

[7] Blasius J, Greenacre M. Visualization of categorical data. San Diego, CA: Academic Press, 1998.

[8] Butler RW, Rorsman I, Hill JM, Tuma R. The effects of frontal brain impairment on fluency: Simple and complex paradigms. Neuropsychology 1993;7:519-29.

[9] Cattell RB. The scree test for the number of factors. Multivariate Behavioral Research 1966;1:245-76.

[10] Chan AS, Butters N, Paulsen JS, Salmon DP, Swenson MR, Maloney LT. An assessment of the semantic network in patients with Alzheimer's disease. Journal of Cognitive Neuroscience 1993;5:254-61.
[11] Chiarello C, Richards L. Another look at categorical priming in the cerebral hemispheres. Neuropsychologia 1992;30:381-92.

[12] Collins AM, Loftus EF. A spreading-activation theory of semantic processing. Psychological Review 1975;82:407-28.

[13] Frith CD, Friston KJ, Herold S, Silbersweig D, Fletcher P, Cahill C, Dolan RJ, Frackowiak RS, Liddle PF. Regional brain activity in chronic schizophrenic patients during the performance of a verbal fluency task. British Journal of Psychiatry 1995; 167:343-9.

[14] Glosser G, Goodglass H. Disorders in executive control functions among aphasic and other brain-damaged patients. Journal of Clinical and Experimental Neuropsychology 1990;12:485501.

[15] Godefroy O, Duhamel A, Leclerc X, Saint Michel T, Henon H, Leys D. Brain-behaviour relationships: Some models and related statistical procedures for the study of brain-damaged patients. Brain 1998;121:1545-56.

[16] Goldberg E, Podell K. Lateralization in the frontal lobes: Searching the right (and left) way. Biological Psychiatry 1995;38:569-71.

[17] Greenacre M, Blasius J. Correspondence analysis in the social sciences: Recent developments and applications. San Diego, CA: Academic Press, 1994.

[18] Gruenewald PJ, Lockhead GR. The free recall of category examples. Journal of Experimental Psychology: Human Learning and Memory 1980;6:225-40.

[19] Kertesz A. Western aphasia battery. New York: Grune \& Stratton, 1982.

[20] Lebart L. Complementary use of correspondence analysis and cluster analysis. In: Greenacre M, Blasius J, editors. Correspondence analysis in the social sciences: Recent developments and applications. San Diego, CA: Academic Press, 1994:162-78.

[21] Lebart L, Morineau A, Warwick KM. Multivariate descriptive statistical analysis: Correspondence analysis and related techniques for large matrices. New York: Wiley, 1984.

[22] Marshall GR, Cofer CN. Single-word free-association norms for 328 responses from the Connecticut Cultural Norms for verbal items in categories. In: Postman L, Keppel G, editors. Norms in word association. New York: Academic Press, 1970:321-60.

[23] Martin A, Wiggs CL, Ungerleider LG, Haxby JV. Neural correlates of category-specific knowledge. Nature 1996;379:649-52.

[24] Milner B. Aspects of human frontal lobe function. In: Jasper $\mathrm{HH}$, Riggio S, Goldman-Rakic PS, editors. Epilepsy and the functional anatomy of the frontal lobe. New York: Raven Press, 1995:67-84.

[25] Mummery CJ, Patterson K, Hodges JR, Wise RJ. Generating 'tiger' as an animal name or a word beginning with T: Differences in brain activation. Proceedings of the Royal Society of London (B) 1996;263:989-95.

[26] Nakagawa A. Role of anterior and posterior attention networks in hemispheric asymmetries during lexical decisions. Journal of Cognitive Neuroscience 1991;3:313-21.

[27] Ramier A-M, Hécaen H. Rôle respectif des atteintes frontales et de la latéralisation lésionnelle dans les déficits de la 'fluence verbale'. Revue Neurologique 1970;123:17-22.

[28] Schiffman SS, Lance Reynolds M, Young FW. Introduction to multidimensional scaling: Theory, methods, and applications. Orlando, FL: Academic Press, 1981.

[29] Spitzer M, Kischka U, Gueckel F, Bellemann ME, Kammer T, Seyyedi S, Weisbrod M, Schwartz A, Brix G. Functional magnetic resonance imaging of category-specific cortical activation: Evidence for semantic maps. Cognitive Brain Research 1998;6:309-19.

[30] Taylor KI, Brugger P, Weniger D, Regard M. Qualitative hemispheric differences in semantic category matching. Brain and Language 1999; 70:119-31. 
[31] Tranel D, Damasio H, Damasio AR. A neural basis for the retrieval of conceptual knowledge. Neuropsychologia 1997; 35:1319-27.

[32] Troster AI, Fields JA, Testa JA, Paul RH, Blanco CR, Hames KA, Salmon DP, Beatty WW. Cortical and subcortical influences on clustering and switching in the performance of verbal fluency tasks. Neuropsychologia 1998;36:295-304.
[33] Troyer AK, Moscovitch M, Winocur G, Alexander MP, Stuss D. Clustering and switching on verbal fluency: The effects of focal frontal- and temporal-lobe lesions. Neuropsychologia 1998;36:499-504.

[34] Tversky A. Features of similarity. Psychological Review 1977;84:327-52. 